\title{
AMPERE polar cap boundaries
}

\author{
Angeline G. Burrell ${ }^{1}$, Gareth Chisham ${ }^{2}$, Stephen E. Milan ${ }^{3}$, Liam Kilcommons ${ }^{4}$, Yun-Ju Chen ${ }^{5}$, Evan G. Thomas ${ }^{6}$, \\ and Brian Anderson ${ }^{7}$ \\ ${ }^{1}$ Space Science Division, U.S. Naval Research Laboratory, 4555 Overlook Ave. SW, Washington, DC, USA \\ ${ }^{2}$ British Antarctic Survey, Cambridge, UK \\ ${ }^{3}$ Radio and Space Plasma Physics, Department of Physics and Astronomy, University of Leicester, University Road, \\ Leicester, UK \\ ${ }^{4}$ Ann and H.J. Smead Department of Aerospace Engineering Sciences, University of Colorado Boulder, 2055 Regent Drive, \\ Boulder, CO, USA \\ ${ }^{5}$ Center for Space Sciences, Department of Physics, The University of Texas at Dallas, 800 West Campbell Road, \\ Richardson, TX, USA \\ ${ }^{6}$ Thayer School of Engineering, Dartmouth College, 14 Engineering Drive, Hanover, NH, USA \\ ${ }^{7}$ Johns Hopkins University Applied Physics Laboratory, 11100 Johns Hopkins Road, Laurel, MD, USA
}

Correspondence: Angeline G. Burrell (angeline.burrell@ nrl.navy.mil)

Received: 2 August 2019 - Discussion started: 21 August 2019

Revised: 21 January 2020 - Accepted: 11 March 2020 - Published: 8 April 2020

\begin{abstract}
The high-latitude atmosphere is a dynamic region with processes that respond to forcing from the Sun, magnetosphere, neutral atmosphere, and ionosphere. Historically, the dominance of magnetosphere-ionosphere interactions has motivated upper atmospheric studies to use magnetic coordinates when examining magnetosphere-ionospherethermosphere coupling processes. However, there are significant differences between the dominant interactions within the polar cap, auroral oval, and equatorward of the auroral oval. Organising data relative to these boundaries has been shown to improve climatological and statistical studies, but the process of doing so is complicated by the shifting nature of the auroral oval and the difficulty in measuring its poleward and equatorward boundaries.

This study presents a new set of open-closed magnetic field line boundaries (OCBs) obtained from Active Magnetosphere and Planetary Electrodynamics Response Experiment (AMPERE) magnetic perturbation data. AMPERE observations of field-aligned currents (FACs) are used to determine the location of the boundary between the Region 1 (R1) and Region 2 (R2) FAC systems. This current boundary is thought to typically lie a few degrees equatorward of the OCB, making it a good candidate for obtaining OCB locations. The AMPERE R1-R2 boundaries are compared to the Defense Meteorological Satellite Program Special Sen-
\end{abstract}

sor J (DMSP SSJ) electron energy flux boundaries to test this hypothesis and determine the best estimate of the systematic offset between the R1-R2 boundary and the OCB as a function of magnetic local time. These calibrated boundaries, as well as OCBs obtained from the Imager for Magnetopauseto-Aurora Global Exploration (IMAGE) observations, are validated using simultaneous observations of the convection reversal boundary measured by DMSP. The validation shows that the OCBs from IMAGE and AMPERE may be used together in statistical studies, providing the basis of a long-term data set that can be used to separate observations originating inside and outside of the polar cap.

\section{Introduction}

The high-latitude atmosphere is a dynamic region with processes that respond to forcing from the Sun, magnetosphere, neutral atmosphere, and ionosphere. The dominant coupling occurs between the ionosphere, magnetosphere, and the solar wind. Interactions between the interplanetary magnetic field (IMF), the magnetic field carried by the solar wind, and the terrestrial magnetosphere result in magnetic reconnection. This creates an area of open field lines (field lines that originate at Earth and connect to the IMF) known as the polar 
cap. The physical processes that occur here are different from those that occur at other high-latitude regions where the magnetic field lines are closed (connect back to the Earth in the opposite hemisphere). In the polar cap, magnetic field lines are moved from magnetic noon to magnetic midnight by the solar wind, where they eventually reconnect with geomagnetic field lines from the opposite hemisphere. Once closed, these field lines move to lower magnetic latitudes (the auroral oval) and return towards the dayside. This process of reconnection is known as the Dungey cycle (Dungey, 1961), and (to first order) describes the motion of the magnetic field lines and the ionospheric plasma frozen into those field lines.

At ionospheric altitudes, the open-closed field line boundary (OCB) separates the polar cap from the auroral oval, which is the highest latitude region to have closed magnetic field lines. This boundary is important because the state of the field lines (open or closed) determines the types of coupling that may occur within the magnetosphere-ionospherethermosphere (MIT) system. One example of a difference in MIT coupling between the polar cap and auroral oval is fieldaligned currents (FACs). The closed field lines in the auroral oval support the formation of current systems that link the ionosphere to the magnetopause and current sheet (the Region 1 or R1 FAC system) and to the partial ring current in the inner magnetosphere (the Region 2 or R2 FAC system) (Iijima and Potemra, 1976). Because the R1 FAC system connects the ionosphere to the outer magnetosphere, it lies poleward of the R2 FAC system and moves with the OCB (Coxon et al., 2018, and references therein).

Another example of MIT coupling processes affected by the OCB is the density structure of the high-latitude ionosphere. Consider the unexceptional case of a southward IMF and a partially illuminated high-latitude ionosphere. Under these conditions, ionospheric plasma follows a convective flow driven by the Dungey cycle, characterised by straight, anti-sunward plasma drifts within the polar cap and longer, curved, sunward drifts when the plasma are frozen into closed magnetic field lines (the boundary between these two regions is commonly referred to as the convection reversal boundary or CRB). The difference in convective motion poleward and equatorward of the CRB creates a highly structured polar ionosphere, as the dense dayside ionospheric plasma is rapidly transported to the nightside where recombination processes destroy plasma that does not return to sunlit regions quickly enough (due to having to follow the longer return path through the auroral oval) (e.g. Spiro et al., 1978).

Due to these and other differences in MIT coupling processes in the auroral oval and the polar cap, it is desirable to have a coordinate system that indicates where (in which region) measurements were taken. This type of adaptive, highlatitude gridding has been performed with various data sets (Redmon et al., 2010; Chisham, 2017b; Kilcommons et al., 2017). These studies have demonstrated improved statistical and climatological results (for example, Chisham (2017b) demonstrated the difference between using magnetic and
OCB-oriented coordinates when studying the climatological behaviour of the plasma drift vorticity) when using adaptive, high-latitude coordinates. Unfortunately, observations of the OCB are sparse. Long-term and large-scale studies would benefit from specifications of the OCB in both hemispheres and all magnetic local times (MLTs) every $15 \mathrm{~min}$ or less (Cowley and Lockwood, 1992). Models that have the ability to distinguish between regions with open and closed field lines would also benefit from adaptive, high-latitude coordinates (Zhu et al., 2019).

This study presents a new set of OCBs obtained from the Active Magnetosphere and Planetary Electrodynamics Response Experiment (AMPERE) magnetic perturbation observations. AMPERE measurements of FACs make it possible to estimate the location where Region 1 (R1) and Region 2 (R2) FAC systems meet (the R1-R2 boundary). Because the location of the Birkeland current system is tied to the expansion and contraction of the polar cap under quiescent and disturbed conditions (Coxon et al., 2018, and references therein.), it seems logical to hypothesise that a dependable relationship between the R1-R2 boundary and the OCB exists. This study investigates the relationship between the AMPERE R1-R2 boundary and the OCB inferred from particle precipitation measurements made by the Defense Meteorological Satellite Program Special Sensor J (DMSP SSJ) electron energy flux boundaries. This study has parallels with that of Clausen et al. (2013), who compared the R1 peak location (as determined from a circle fitted to the R1 peaks at all MLTs) with a range of different DMSP particle precipitation boundaries, showing a close relationship with the b5i and b5e boundaries in the nightside ionosphere. Section 2 presents the details of both data sets. Section 3 explores the relationship between the different boundaries and presents the calibration process that allows the AMPERE R1-R2 boundary to be used as a proxy for the OCB. This calibration, as well as the previous Magnetopause-to-Aurora Global Exploration (IMAGE) calibration performed by Chisham (2017b), is validated in Sect. 4 by comparing calibrated OCBs with the CRBs from DMSP plasma drift measurements. CRBs were chosen as a validation data set because the direction of convective plasma drifts are strongly tied to the motion and state (i.e. open or closed) of the magnetic field lines. This means that the CRB is typically located at or just equatorward of the OCB (Newell et al., 2004; Drake et al., 2009), except for regions of the dayside and nightside ionosphere that map to regions of ongoing magnetic reconnection. Finally, the results of this study are summarised in Sect. 5 .

\section{Instrumentation}

The data sets used in this study have a long and ongoing history of observations. The primary data set, AMPERE, is described in Sect. 2.1. Two instruments from DMSP are used, one for calibration of the boundaries and another for valida- 
tion. Both DMSP data sets are described in Sect. 2.2. The IMAGE far ultraviolet (FUV) data set used in the validation is described in Sect. 2.3.

\subsection{AMPERE}

AMPERE assimilates measurements from the approximately 70 polar-orbiting spacecraft of the Iridium telecommunications constellation to deduce the high-latitude distribution of horizontal magnetic field perturbations produced by the FACs responsible for magnetosphere-ionosphere coupling (Anderson et al., 2000, 2002; Waters et al., 2001; Coxon et al., 2018). The FAC pattern in both hemispheres is calculated from $10 \mathrm{~min}$ averages at a 2 min cadence on a magnetic latitude and MLT grid $\left(1^{\circ} \times 1 \mathrm{~h}\right.$ resolution); this study employs R1-R2 FAC boundaries from 2010 to 2012 (Milan, 2019).

The basis of the R1-R2 boundary identification is a fitting technique described by Milan et al. (2015). This technique aims to determine the centre and radius of the circle that best describes the boundary between the R1 and R2 FACs without fitting to individual MLT bins. By avoiding this common method of defining a high-latitude boundary, this R1R2 boundary identification is more robust in the event of sparse or weak currents and less influenced by the poorly defined current structures near local magnetic noon and midnight.

The following procedure is applied to each AMPERE FAC grid. In this description, positive and negative values represent upward and downward currents respectively. The R1 currents flow upwards at dusk and downwards at dawn, whereas the R2 currents have the opposite polarity and lie equatorward of the R1 current system. To distinguish between these two FAC systems, the first step is to multiply all FAC magnitudes on the dawn side (00:00 $\leq$ MLT $<12: 00)$ by -1 . This redefines the current signs such that R1 FACs are positive and R2 FACs are negative at all MLTs. Then a centre point $\left(x_{0}, y_{0}\right)$ is assumed, where $x_{0}$ is the dawnward distance from the noon-midnight meridian and $y_{0}$ is the sunward distance from the dawn-dusk meridian. A range of centres are tested, with $x_{0}$ varying between $\pm 4^{\circ}$ and $y_{0}$ varying between -6 and $0^{\circ}$ latitude. Additionally, a range of radii are tested at each centre point; the radius is varied by $1^{\circ}$ latitude $(111 \mathrm{~km})$ from 8 to $35^{\circ}$. At each radius and centre point, the sum of the FACs at 200 equally spaced points in a ring centred at $\left(x_{0}\right.$, $y_{0}$ ) is found. This produces a profile of integrated FAC magnitude with the radius, in which a negative-positive bipolar signature is sought. The zero-crossing of the bipolar signature is taken to be the R1-R2 boundary, and the peak-to-peak magnitude provides a figure of merit (FOM) for the boundary fit. For each AMPERE FAC grid, the circle with the best FOM is chosen, and grids with low FOMs are discarded as being unreliable.

\subsection{DMSP}

The DMSP OCB locations are obtained from energetic electron fluxes measured by three DMSP spacecraft (F16-F18) that were operational and have updated ephemera (Redmon et al., 2017) during the period of time when AMPERE R1-R2 boundaries were available. The DMSP satellites were located in sun-synchronous polar orbits at an altitude of about $830 \mathrm{~km}$, with an orbital period of approximately $101 \mathrm{~min}$. The geographic locations of the DMSP SSJ/5 equatorward and poleward boundaries were determined using ssj_auroral_boundary (Kilcommons and Burrell, 2019), which implements the technique described in Kilcommons et al. (2017). A clean set of OCBs were obtained by selecting the poleward boundaries with figures of merit greater than 3.0 and calculating the AACGM-v2 coordinates at each location (Shepherd, 2014; Burrell et al., 2018b).

The same DMSP spacecraft also carry an Ion Velocity Meter (IVM) that measures the three-dimensional ion velocity (Heelis and Hanson, 1998). As discussed in Sect. 1, the CRB is the location where plasma drifts change from moving sunward to anti-sunward, or vice versa, and this boundary typically lies at or just equatorward of the OCB (Newell et al., 2004; Drake et al., 2009).

In this paper, CRBs obtained by Chen et al. (2015) are used to validate the AMPERE OCB locations within an hour of dawn (06:00 MLT) and dusk (18:00 MLT). Other MLTs were not considered for several reasons. Most importantly,

1. near magnetic noon and midnight the flows tend to be mostly sunward or anti-sunward, meaning there is no clear reversal in the convection as a function of magnetic latitude;

2. the IMF orientation will shift the MLT location of these sunward or anti-sunward flows, meaning more local times than just noon and midnight are affected; and

3. near midnight, the Harang reversal can give the appearance of multiple convection reversals at different latitudes.

The Chen et al. (2015) algorithm is optimised to identify the CRB in a two-cell convection pattern. If the plasma convection has a complex pattern with more than four reversals, or the plasma flows are weak and noisy, the program will not identify any CRB location. For symmetric, multicell patterns (such as those observed when the IMF is dominated by a positive $B_{Z}$ component), the program will identify the most equatorward reversal boundary. Otherwise, the most poleward reversal boundary will be selected as the CRB location. The algorithm typically performs better in the summer, as the DMSP IVM performs better when the plasma density is higher (Chen et al., 2015; Chen and Heelis, 2018). These algorithmic biases mean that the CRBs cover May through August in the Northern Hemisphere and November through 
February in the Southern Hemisphere. However, even with the difficulties introduced by nonsymmetric convection patterns, all IMF clock angles are well represented in the CRB data set.

\subsection{IMAGE FUV}

Chisham (2017b) obtained estimates of the OCB from auroral images measured by the FUV imagers onboard the IMAGE spacecraft. Images of the Northern Hemisphere auroral region were available for the epoch spanning from May 2000 to August 2002. During this time, the spacecraft was located in an elliptical orbit with a $90^{\circ}$ inclination, an apogee of $7 R_{\mathrm{E}}$, a perigee of $1000 \mathrm{~km}$, and an orbital period of $\sim 13.5 \mathrm{~h}$.

This study uses data from the two FUV spectrographic imagers, SI12 and SI13 (Mende et al., 2000). The SI13 imager measured oxygen emissions at $135.6 \mathrm{~nm}$, resulting from energetic electron precipitation. The SI12 imager measured Doppler-shifted Lyman- $\alpha$ emissions at $121.8 \mathrm{~nm}$, resulting from proton precipitation. Both imagers provided data at a 2 min resolution, when the Northern Hemisphere was visible. The OCB was identified in the individual FUV images and fit across all magnetic local times using the techniques described by Longden et al. (2010) and Chisham (2017b).

\section{Relationship between the R1-R2 boundary and OCB}

This study follows the process outlined in Boakes et al. (2008), which determined the offset between the IMAGE FUV poleward auroral boundaries and DMSP OCBs, to obtain a correction between the AMPERE R1-R2 boundary and the DMSP SSJ OCBs. The five steps of this process are enumerated in the following:

1. identify the AMPERE R1-R2 boundaries;

2. pair AMPERE R1-R2 boundaries with DMSP SSJ OCBs;

3. determine the typical offset at different MLTs;

4. find a functional fit that describes the offset between the DMSP SSJ OCBs and the AMPERE R1-R2 boundaries; and

5. use the functional fit to correct the AMPERE R1-R2 boundary locations, creating an AMPERE OCB proxy.

This study uses AMPERE R1-R2 boundaries, described in Sect. 2.1, from January 2010 through December 2012. Using only R1-R2 boundaries with FOMs greater than $0.15 \mathrm{~mA}$ provides 636250 Northern Hemisphere and 531666 Southern Hemisphere boundary locations. Pairing these boundaries to good DMSP SSJ OCB detections by requiring each observation be taken within $10 \mathrm{~min}$ of each other leaves 29683 Northern Hemisphere and 29135 Southern Hemisphere boundaries. The $10 \mathrm{~min}$ window for pairing boundaries was chosen because of the $10 \mathrm{~min}$ averaging performed on the AMPERE FAC maps (see Sect. 2.1). However, over $90 \%$ of Northern Hemisphere pairs and over $80 \%$ of Southern Hemisphere pairs have a temporal difference of 1 min or less. Good DMSP SSJ OCB detections are defined as having a FOM of 3.0 or greater. This is consistent with the work presented by Kilcommons et al. (2017) and reduces the number of passes with dayside precipitation associated with the cusp, mantle, and other sources whose origin (inside or outside the polar cap) is still debatable. The DMSP SSJ paired OCBs for each hemisphere and satellite are shown in Fig. 1 as a scatter plot, with the median location of the AMPERE R1-R2 boundaries plotted on top. Note that the R1-R2 boundaries lie near the equatorward edge of the DMSP SSJ OCBs. Because of the DMSP satellite orbits, MLTs near noon are only covered in the Northern Hemisphere and those near midnight are only covered in the Southern Hemisphere.

Ideally, observations from both hemispheres can be combined to provide complete MLT coverage of the differences between the AMPERE R1-R2 boundaries and DMSP SSJ OCBs. To test the assumption that the northern and southern boundaries have the same local time dependence, the MLT bins with observations in both hemispheres (05:00 08:00 and 15:00-20:00 MLT) were compared. The hourly boundary offsets in each hemisphere and both hemispheres combined, all calculated using the magnetic co-latitude, are presented in Table 1.

The boundary offsets in Table 1 were calculated by finding the typical difference between the DMSP SSJ OCB and the AMPERE R1-R2 boundary location in AACGM-v2 magnetic latitude in $1 \mathrm{~h}$ MLT bins. The typical boundary latitude difference ( $\Delta \phi$, which equals the DMSP SSJ OCB colatitude minus the AMPERE R1-R2 boundary co-latitude) is represented by two values - the median of the boundary latitude differences and the peak of a Gaussian distribution (S.G. peak) - fitted to a smoothed histogram (as in Boakes et al., 2008). The histograms have $1^{\circ}$ bins, and they were smoothed using a $4^{\circ}$ running average. The smoothed histogram was then fitted with a Gaussian function, allowing the S.G. peak and standard deviation to be calculated.

Comparing the median and S.G. peak of the $\Delta \phi$ for the MLT bins with observations in both hemispheres shows a mean hemispheric difference of -0.30 and $0.23^{\circ}$ for the median and S.G. peaks respectively. This difference is small enough to justify combining the northern and southern hemispheric $\Delta \phi$, as it is much smaller than the mean standard deviation of the MLT distributions ( $\bar{\sigma}=2.66^{\circ}$ for the overlapping MLT bins). The results for the combined hemispheres are presented in the rightmost columns of Table 1 and in Fig. 2. There is about a $0.49^{\circ}$ difference between the median and S.G. peak values. This difference is very small compared with the width of the $\Delta \phi$ distributions, and it provides a measure of uncertainty for the resulting boundary correction.

Unfortunately, the differences between the boundary fitting methodology used by Chisham (2017b) and Milan et al. (2015) mean that it is not reasonable to use a harmonic func- 
Table 1. Hourly boundary offset for hours with over 100 boundary pairs and successfully fit Gaussians.

\begin{tabular}{|c|c|c|c|c|c|c|}
\hline \multirow[t]{2}{*}{ MLT } & \multicolumn{2}{|c|}{ North } & \multicolumn{2}{|c|}{ South } & \multicolumn{2}{|c|}{ Both } \\
\hline & Median $\left(^{\circ}\right)$ & S.G. peak $\left(^{\circ}\right)$ & $\operatorname{Median}\left({ }^{\circ}\right)$ & S.G. peak $\left(^{\circ}\right)$ & Median $\left({ }^{\circ}\right)$ & S.G. peak $\left({ }^{\circ}\right)$ \\
\hline 00:00 & - & - & 2.04 & 2.83 & 2.04 & 2.83 \\
\hline 01:00 & - & - & 1.88 & 2.56 & 1.88 & 2.56 \\
\hline 02:00 & - & - & 1.93 & 2.36 & 1.93 & 2.36 \\
\hline 03:00 & - & - & 2.46 & 2.94 & 2.46 & 2.94 \\
\hline 04:00 & - & - & 3.20 & 3.60 & 3.20 & 3.60 \\
\hline 05:00 & 3.96 & 4.45 & 4.80 & 5.29 & 4.33 & 4.86 \\
\hline 06:00 & 5.16 & 5.69 & 6.34 & - & 5.73 & 6.26 \\
\hline 07:00 & 5.29 & 5.88 & 6.98 & - & 6.21 & 6.71 \\
\hline 08:00 & 5.69 & 6.19 & 7.10 & - & 6.08 & 6.64 \\
\hline 09:00 & 5.38 & 5.99 & - & - & 6.35 & 6.88 \\
\hline 10:00 & 4.64 & 5.29 & - & - & 5.64 & 6.23 \\
\hline 11:00 & 3.78 & 4.27 & - & - & 3.82 & 4.32 \\
\hline $12: 00$ & 3.57 & 3.99 & - & - & 3.66 & 4.04 \\
\hline 13:00 & 3.30 & 3.61 & - & - & 3.40 & 3.62 \\
\hline $14: 00$ & 2.95 & 3.36 & - & - & 3.02 & 3.43 \\
\hline $15: 00$ & 3.49 & 3.97 & 5.21 & 5.76 & 3.97 & 4.50 \\
\hline $16: 00$ & 4.20 & 4.68 & 4.19 & 4.66 & 4.19 & 4.67 \\
\hline $17: 00$ & 4.00 & 4.47 & 3.32 & 3.74 & 3.77 & 4.22 \\
\hline 18:00 & 2.82 & 3.30 & 2.27 & 2.77 & 2.54 & 3.01 \\
\hline $19: 00$ & 2.67 & 3.12 & 1.52 & 1.95 & 2.07 & 2.51 \\
\hline 20:00 & 2.42 & 3.13 & 0.96 & 1.35 & 1.29 & 1.63 \\
\hline 21:00 & - & - & 0.33 & 0.73 & 0.33 & 0.73 \\
\hline $22: 00$ & - & - & 0.14 & 0.60 & 0.14 & 0.60 \\
\hline 23:00 & - & - & 1.24 & 1.94 & 1.24 & 1.94 \\
\hline
\end{tabular}

Table 2. Boundary fit constants for DMSP - AMPERE boundary offset.

\begin{tabular}{lrr}
\hline Constant & Median & S.G. peak \\
\hline$a$ & $4.01^{\circ}$ & $4.41^{\circ}$ \\
$e$ & 0.55 & 0.51 \\
$\tau$ & -0.92 & -0.95 \\
\hline
\end{tabular}

tion to describe the offset between the DMSP SSJ OCBs and the AMPERE R1-R2 boundaries, as done in prior auroral boundary fitting studies (Holzworth and Meng, 1975; Carbary et al., 2003; Boakes et al., 2008). Because the R1-R2 boundary fitting method used by Milan et al. (2015) does not fit a series of MLT bins, the boundary correction cannot be applied prior to circle fitting and will determine the final shape of the OCB proxy. Thus, this study uses a generalised ellipse (Eq. 1) rather than a harmonic function to avoid overfitting the MLT dependence of the offset between the DMSP SSJ OCBs and the AMPERE R1-R2 boundaries.

$K(\lambda)=\frac{a\left(1-e^{2}\right)}{1+e \cos (\lambda-\tau)}$

In Eq. (1), $\lambda$ is the MLT in radians, $a$ is the semi-major axis in degrees, $e$ is the eccentricity (a unitless quantity), and $\tau$ is the angular offset of the ellipse's centre in radians. These four constants allow the ellipse to adjust its centre and axes. They are fit using the Python SciPy least squares fitting routine, leastsq (Virtanen et al., 2020), which wraps the MINPACK LMDIF and LMDER algorithms (More et al., 1984). The least squares fitting routine minimises the difference between $K$ and $\Delta \phi$, weighted by the inverse of the error, $\epsilon$. The error is defined as shown in Eq. (2), where $N_{\text {MLT }}$ is the number of $\Delta \phi$ observations in each MLT bin, $N_{\max }$ is the maximum $N_{\mathrm{MLT}}$, and $\sigma$ is either the interquartile range or the standard deviation depending on whether the median or S.G. peak was used as the central value. The results of this fitting procedure are shown in Fig. 3 and Table 2.

$\epsilon=\sqrt{\left(\frac{N_{\mathrm{MLT}}}{N_{\max }}\right)^{2}+\sigma^{2}}$

As shown in Fig. 3, the AMPERE R1-R2 boundary lies about $2^{\circ}$ equatorward of the OCB at magnetic midnight, about $4^{\circ}$ equatorward of the OCB at magnetic noon, and further out at dawn and dusk. The elliptical fit follows the central values very closely between 00:00 and 10:00 MLT, and it smooths through the maxima and minima at 12:00, 16:00, and 22:00 MLT. However, even where the differences are greatest, the elliptical fit does not differ from the central value by more than $\epsilon / 2$. This behaviour is consistent whether 


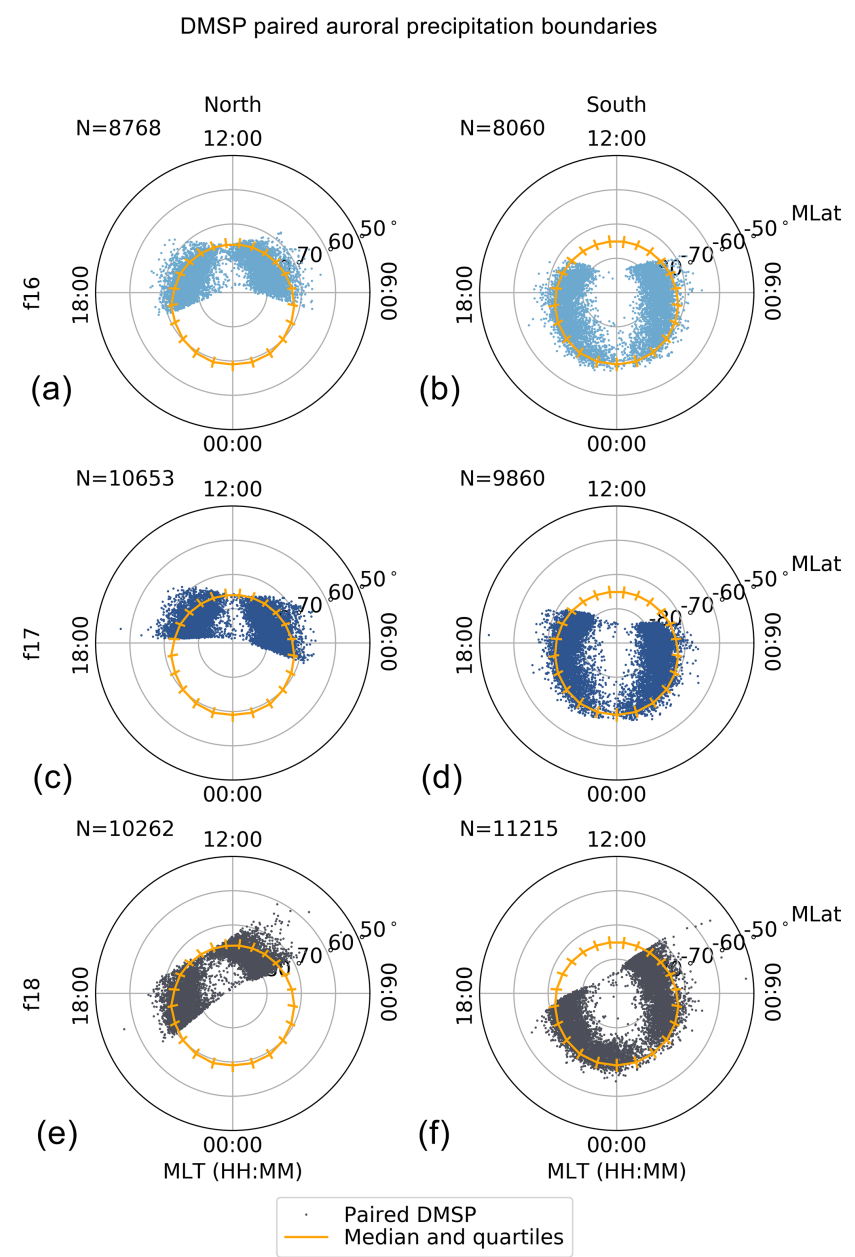

Figure 1. Paired AMPERE R1-R2 boundaries and DMSP SSJ OCBs for both hemispheres (Northern Hemisphere is shown in a, c, and $\mathbf{e}$; Southern Hemisphere is shown in, $\mathbf{b}, \mathbf{d}$, and $\mathbf{f}$ ) and each satellite. The scattered points show the DMSP SSJ OCBs, and the gold circle shows the median location of the AMPERE R1-R2 boundaries. The scatter bars denote the quartiles of the paired AMPERE $\mathrm{R} 1-\mathrm{R} 2$ boundaries.

the median or S.G. peak is used in the fitting process. The similarity between the two fits can be quantified by comparing the differences between $a_{\text {Median }}$ and $a_{\text {S.G. Peak }}\left(0.40^{\circ}\right)$ and the typical difference between the hourly median and S.G. peak values $\left(0.49^{\circ}\right)$; the differences between the eccentricity and angular offset are even less significant.

The consistency of the elliptical fit for both central values, as well as its success at capturing the major features of $\Delta \phi$ given the functional constraints, make it a good candidate for correcting the R1-R2 boundary to provide an OCB estimate. The Gaussian nature of the hourly bins (shown in Fig. 2) suggests that differences between the R1-R2 boundary and DMSP SSJ OCB are randomly distributed, confirming the conclusion that it is appropriate to use $K$ to correct the R1-R2 boundary to obtain an AMPERE OCB estimate.

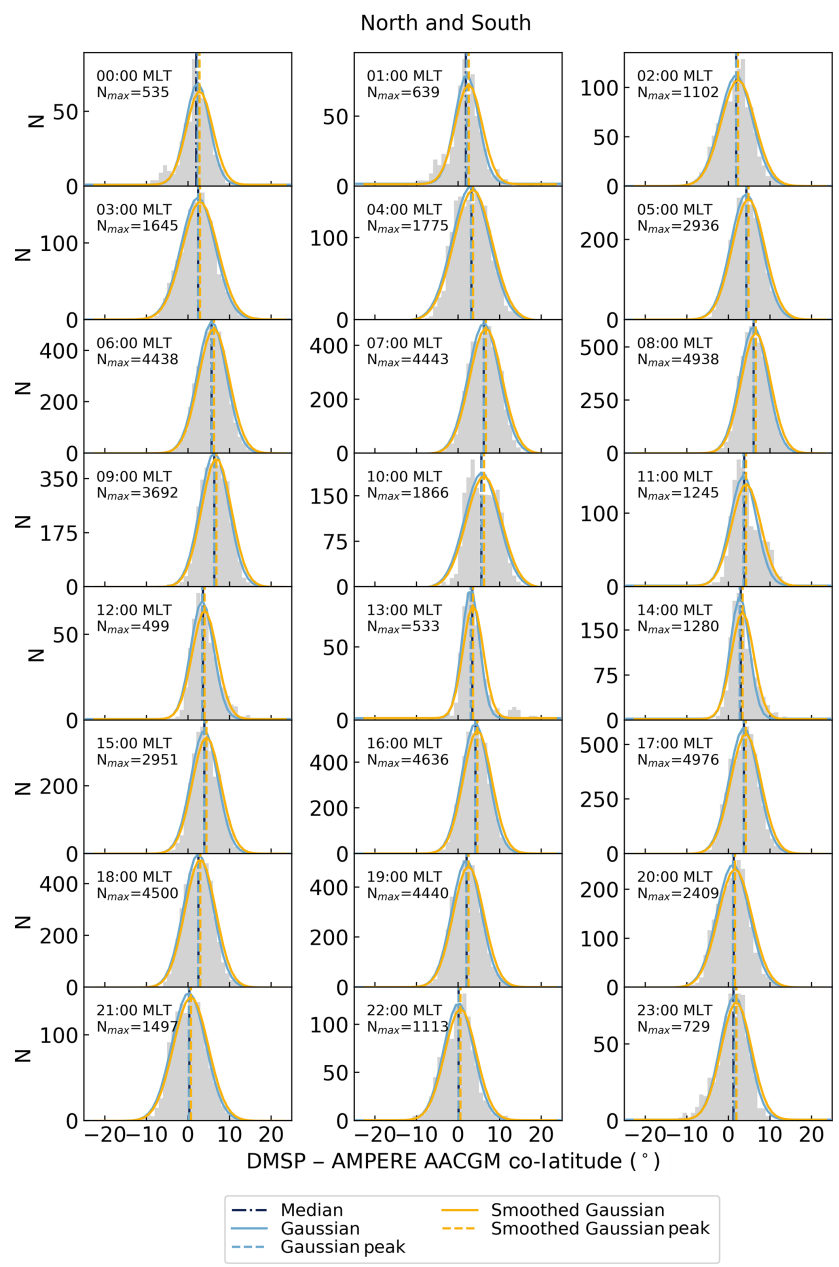

Figure 2. Hourly distributions of paired AMPERE R1-R2 boundary and DMSP SSJ OCB latitude differences, with boundary differences from both hemispheres and all satellites. The black dashed line shows the median of the distribution, the blue line shows a Gaussian fit to the distribution and the gold line shows the Gaussian fit to the smoothed histogram. The vertical blue and gold lines show the peaks of each Gaussian fit.

\section{Validation}

The appropriateness of using $K$ to transform the AMPERE R1-R2 boundary into an AMPERE OCB is tested by comparing the AMPERE OCBs to the DMSP CRBs within an hour of dawn and dusk. These local times were chosen due to the MLT-dependent variations in the CRB-OCB relationship discussed in Sect. 2.2. It should also be reiterated that no specific selection was made for IMF conditions. All IMF clock angles and magnitudes are considered together, as the AMPERE OCBs should be valid at all IMF conditions when the OCB can be represented (to first order) by an ellipse. To ensure that the performance of the AMPERE OCBs are on par with previous OCB calculations, this validation is also performed for the IMAGE OCBs. Unfortunately, it is impos- 


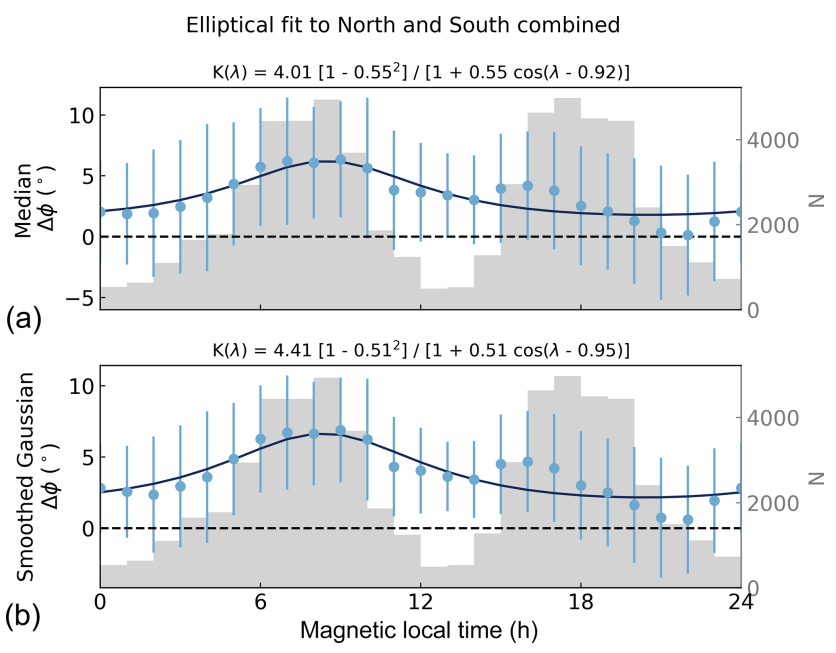

Figure 3. Elliptical boundary correction (black line) fit to the median (a) and S.G. peak (b) $\Delta \phi$ for both hemispheres. The blue dots and scatter bars show the central value and $\epsilon$ in each MLT bin respectively. The grey histogram shows $N_{\mathrm{MLT}}$, and scales to the $y$ axis on the right.

sible to directly compare the AMPERE and IMAGE OCBs because there is no temporal overlap between the two data sets. This validation effort paired OCBs with DMSP CRBs that were identified within 10 min of one another. The location of the DMSP CRB relative to the OCB was then determined. In this adaptive coordinate system, the OCB is set at a co-latitude of $74^{\circ}$ (a latitude chosen to represent the OCB in adaptive, high-latitude coordinates based on the typical size of the polar cap). CRBs that occur poleward or equatorward of the OCB will have co-latitudes greater than or less than $74^{\circ}$ respectively. This adaptive gridding was performed using the ocbpy Python package (Burrell and Chisham, 2018; Burrell et al., 2018a).

Figure 4 shows the distribution of CRB observations for the different DMSP satellites, OCB sources, and hemispheres. As was done with the DMSP SSJ observations, 2 years of CRBs and OCBs were paired in time after removing unreliable boundaries (as discussed in Sect. 2). Note that the paired data, both from the two IMAGE instruments and from AMPERE (in both hemispheres), show a similar spread of CRBs at different magnetic local times, with larger spreads near magnetic noon and midnight.

Figure 5 shows the histograms of the latitude differences between the DMSP CRBs and the IMAGE (Fig. 5a, d) or AMPERE (Fig. 5b, c, e, f) OCBs. This figure also shows the results for the median ellipse correction to obtain the AMPERE OCB (Fig. 5a, b, c) and the S.G. peak ellipse correction (Fig. d, e, f). For the IMAGE histograms, Fig. 5a shows the results for the SI13 instrument, and Fig. 5d shows the results for the SI12 instrument. In all cases, the means and medians of the difference distributions behave similarly: most points lie within $1^{\circ}$ of each other, and the standard deviation

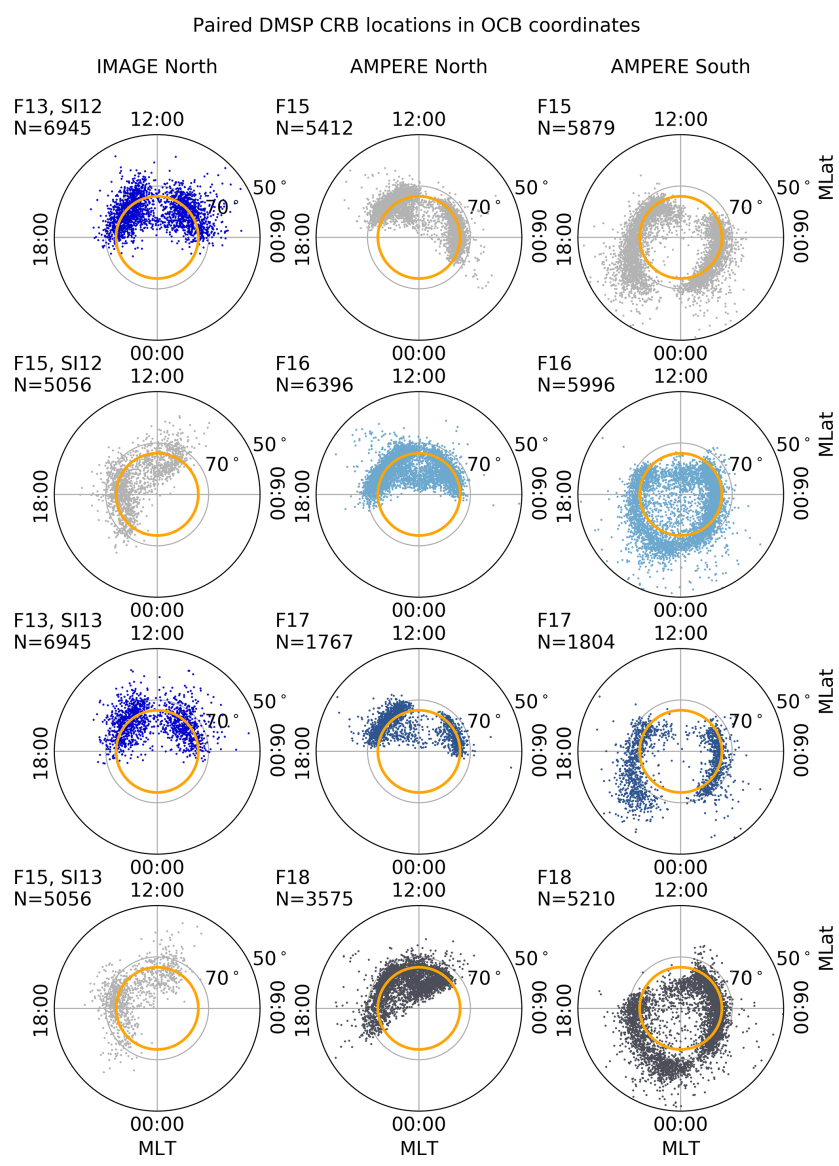

Figure 4. Paired IMAGE and AMPERE OCBs with DMSP CRBs for the available hemispheres and each satellite. The IMAGE data show the SI12 and SI13 observations for the Northern Hemisphere (left column), while the median elliptical correction was applied to obtain the AMPERE OCBs shown in the middle and right columns (which show the Northern Hemisphere and Southern Hemisphere respectively). The scattered points show the DMSP IVM CRBs, and the gold circle shows the IMAGE or AMPERE OCB. To simplify the comparison, the DMSP IVM CRB locations are plotted in adjusted polar coordinates (Burrell and Chisham, 2018). Although all CRBs paired with IMAGE or AMPERE OCBs are shown here, only CRBs within $1 \mathrm{~h}$ of 06:00 or 18:00 MLT were used in this validation.

of the distributions is below $5^{\circ}$ in all places. Additionally, the CRB is approximately co-located with both the AMPERE and IMAGE OCBs. This close agreement with the DMSP CRB and the similar behaviour of the IMAGE and AMPERE OCBs validates the AMPERE OCBs provided here.

\section{Conclusions}

This study modified traditional auroral boundary fitting methods to establish an MLT-dependent relationship between the OCB and the R1-R2 boundary. This was performed by determining the first moment of the distribution of differences between the R1-R2 boundary and the OCB (as mea- 
Boundary differences within $1 \mathrm{~h}$ of $18: 00$ or 06:00 MLT
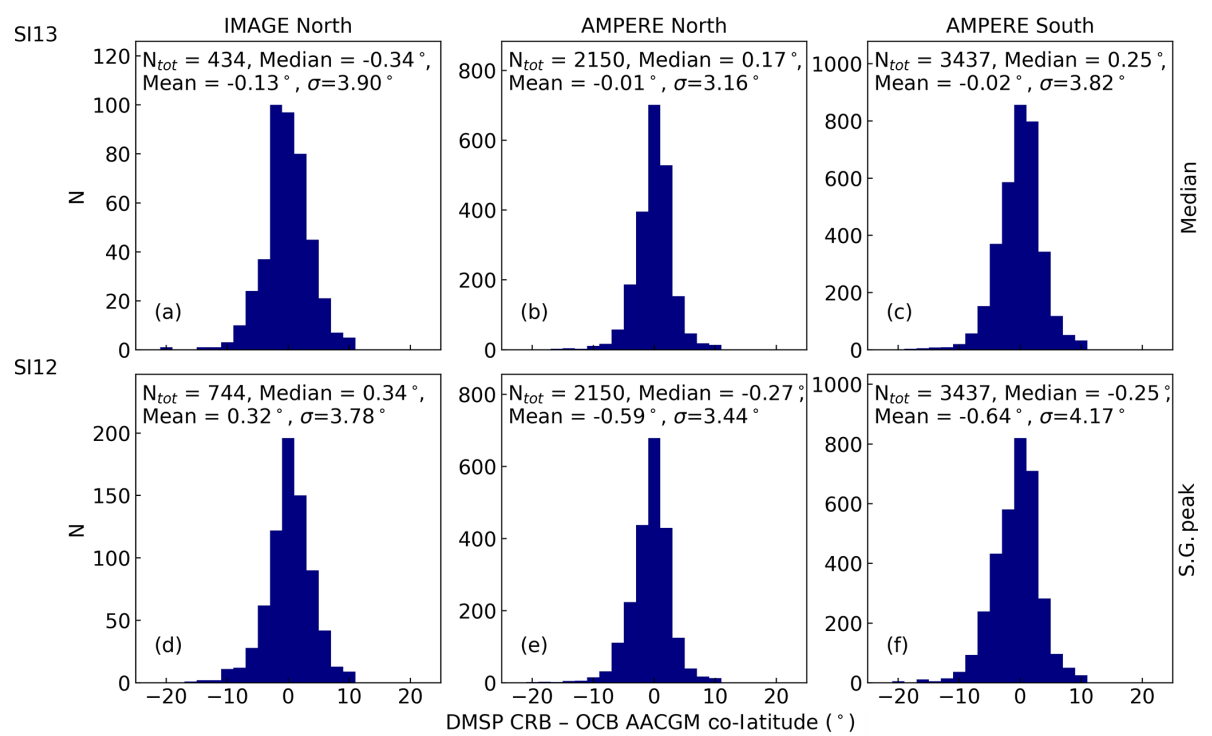

Figure 5. Histograms showing the differences between DMSP CRB and IMAGE or AMPERE OCB using paired boundaries that occur within $1 \mathrm{~h}$ of $06: 00$ or 18:00 MLT.

sured by the DMSP SSJ instrument) for $1 \mathrm{~h}$ MLT bins. These moments (which included the median of the distribution and the peak of a smoothed Gaussian fit) were then used to define the parameters of an elliptical function. This function specifies the distance between the OCB and R1-R2 boundary as a function of MLT.

The validity of this OCB, as well as previously determined IMAGE OCBs, were tested against the dawn and dusk measurements of the CRB (as measured by several DMSP IVM instruments). These boundaries were found to typically differ by less than a degree.

As mentioned in the introduction, modelling and statistical studies in polar regions should avoid mixing measurements taken in the auroral oval and the polar cap. In combination, the AMPERE and IMAGE OCBs form the basis of a multi-solar cycle data set that could be used to improve high-latitude statistical studies and climatological models. The data sets and software tools presented in this paper allow researchers to begin using adaptive, high-latitude coordinates in their investigations.

Code and data availability. AMPERE data are available from the John Hopkins University Applied Physics Laboratory at http:// ampere.jhuapl.edu/ (John Hopkins Applied Physics Laboratory, 2019). We thank the AMPERE team and the AMPERE Science Center for providing the Iridium-derived data products. AMPERE boundaries are described in Milan et al. (2015) and can be accessed at https://doi.org/10.25392/leicester.data.11294861.v1 (Milan, 2019) or through ocbpy (Burrell and Chisham, 2018).

The IMAGE FUV data are provided courtesy of the instrument PI Stephen Mende (University of California, Berkeley).
We thank the PI, the IMAGE mission, and the IMAGE FUV team for data usage and processing tools. The raw IMAGE data and software are available from http://sprg.ssl.berkeley.edu/image/ (Frey, 2017). The auroral boundary data set and the methodology used to create it can be found at https://www.bas.ac.uk/project/ image-auroral-boundary-data/ (last access: August 2019) or in Chisham (2017a).

DMSP data are available at http://cedar.openmadrigal.org (Rideout, 2019), and https://cdaweb.gsfc.nasa.gov (Kovalick, 2019). DMSP SSJ boundaries may be obtained using the software available at https://doi.org/10.5281/zenodo.3267415 (Kilcommons and Burrell, 2019) and https://doi.org/10.5281/zenodo.3373812 (Kilcommons et al., 2019). DMSP CRBs can be requested from YunJu Chen (yxc126130@utdallas.edu).

The software that was used to perform adaptive, high-latitude gridding can be found at https://github.com/aburrell/ocbpy (last access: August 2019) or https://doi.org/10.5281/zenodo.1217177 (Burrell and Chisham, 2018).

Author contributions. AGB developed the concept, performed the data analysis, and wrote the paper. GC supported the conceptual development, provided feedback on the data analysis, and edited the paper. SEM provided the AMPERE R1-R2 boundaries and guidelines for their use, provided feedback on the conceptual development, and contributed to writing the paper. LK provided guidelines for the use of the DMSP SSJ boundaries, gave feedback on the validation, and edited the paper. YJC provided the DMSP CRBs, supplied guidelines for their use in validation, and edited the paper EGT provided feedback on the data validation efforts and edited the paper. BA is the PI of AMPERE. 
Competing interests. The authors declare that they have no conflict of interest.

Acknowledgements. Angeline G. Burrell is supported by the United States Chief of Naval Research (US CNR). Gareth Chisham is supported by United Kingdom Research and Innovation (UKRI) as part of the British Antarctic Survey - Polar Science for Planet Earth Programme. Liam Kilcommons is supported by AFOSR (award no. FA9550-17-1-0258). Yun-Ju Chen is supported by an AFOSR MURI grant (grant no. FA9559-16-1-0364) to The University of Texas at Dallas. Evan G. Thomas is supported by a NSF grant. The authors would like to thank Jone Peter Reistad for communications about the "Discussion Paper" version of this paper that lead to important improvements.

Financial support. This research has been supported by the United States Chief of Naval Research (US CNR); the United Kingdom Research and Innovation (UKRI), British Antarctic Survey - Polar Science for Planet Earth Programme; the Air Force Office of Scientific Research (grant nos. FA9550-17-1-0258 and FA9559-161-0364); and the National Science Foundation, Office of Polar Programs (grant no. OPP-1836426).

Review statement. This paper was edited by Keisuke Hosokawa and reviewed by two anonymous referees.

\section{References}

Anderson, B. J., Takahashi, K., and Toth, B. A.: Sensing global Birkeland currents with Iridium engineering magnetometer data, Geophys. Res. Lett., 27, 4045-4048, 2000.

Anderson, B. J., Takahashi, K., Kamei, T., Waters, C. L., and Toth, B. A.: Birkeland current system key parameters derived from Iridium observations: Method and initial validation results, J. Geophys. Res., 107, 1079, https://doi.org/10.1029/2001JA000080, 2002.

Boakes, P. D., Milan, S. E., Abel, G. A., Freeman, M. P., Chisham, G., Hubert, B., and Sotirelis, T.: On the use of IMAGE FUV for estimating the latitude of the open/closed magnetic field line boundary in the ionosphere, Ann. Geophys., 26, 2759-2769, https://doi.org/10.5194/angeo-26-2759-2008, 2008.

Burrell, A. G. and Chisham, G.: aburrell/ocbpy: Beta Release, Zenodo, https://doi.org/10.5281/zenodo.1217177, 2018.

Burrell, A. G., Halford, A., Klenzing, J., Stoneback, R. A., Morley, S. K., Annex, A. M., Laundal, K. M., Kellerman, A. C., Stansby, D., and Ma, J.: Snakes on a Spaceship-An Overview of Python in Heliophysics, J. Geophys. Res.-Space, 123, 1038410402, https://doi.org/10.1029/2018ja025877, $2018 \mathrm{a}$.

Burrell, A. G., van der Meeren, C., and Laundal, K. M.: aburrell/aacgmv2: AACGMV2 2.5.1, Zenodo, https://doi.org/10.5281/zenodo.1469697, 2018 b.

Carbary, J. F., Sotirelis, T., Newell, P. T., and Meng, C.-I.: Auroral boundary correlations between UVI and DMSP, J. Geophys. Res., 108, 1018, https://doi.org/10.1029/2002JA009378, 2003.
Chen, Y.-J. and Heelis, R. A.: Motions of the Convection Reversal Boundary and Local Plasma in the HighLatitude Ionosphere, J. Geophys. Res.-Space, 123, 2953-2963, https://doi.org/10.1002/2017ja024934, 2018.

Chen, Y.-J., Heelis, R. A., and Cumnock, J. A.: Response of the ionospheric convection reversal boundary at high latitudes to changes in the interplanetary magnetic field, J. Geophys. Res.Space, 120, 5022-5034, https://doi.org/10.1002/2015ja021024, 2015.

Chisham, G.: Auroral Boundary Derived from IMAGE Satellite Mission Data (May 2000-Oct 2002), British Antarctic Survey, https://doi.org/10.5285/75aa66c1-47b4-4344-ab5d52ff2913a61e, 2017a.

Chisham, G.: A new methodology for the development of high-latitude ionospheric climatologies and empirical models, J. Geophys. Res.-Space, 122, 932-947, https://doi.org/10.1002/2016JA023235, 2017b.

Clausen, L. B. N., Baker, J. B. H., Ruohoniemi, J. M., Milan, S. E., Coxon, J. C., Wing, S., Ohtani, S., and Anderson, B. J.: Temporal and spatial dynamics of the regions 1 and 2 Birkeland currents during substorms, J. Geophys. Res.-Space, 118, 30073016, https://doi.org/10.1002/jgra.50288, 2013.

Cowley, S. W. H. and Lockwood, M.: Excitation and decay of solar-wind driven flows in the magnetosphere-ionosphere system, Ann. Geophys., 10, 103-115, 1992.

Coxon, J. C., Milan, S. E., and Anderson, B. J.: A review of Birkeland current research using AMPERE, vol. 235, chap., in: Electric Currents in Geospace and Beyond, American Geophysical Union, https://doi.org/10.1002/9781119324522.ch16, 257-278, 2018.

Drake, K. A., Heelis, R. A., Hairston, M. R., and Anderson, P. C.: Electrostatic Potential Drop Across the Ionospheric Signature of the Low-Latitude Boundary Layer, J. Geophys. Res., 114, A04215, https://doi.org/10.1029/2008JA013608, 2009.

Dungey, J. W.: Interplanetary Magnetic Field and the Auroral Zones, Phys. Rev. Lett., 6, 47-48, 1961.

Frey, H. U.: Image, available at: http://sprg.ssl.berkeley.edu/image/, last access: August 2017.

Heelis, R. A. and Hanson, W. B.: Measurements of Thermal Ion Drift Velocity and Temperature using Planar Sensors, in: Measurement Techniques in Space Plasmas: Particles, edited by: Pfaff, R. F., Borovsky, J., and Young, T. D., AGU, Washington, D.C., https://doi.org/10.1029/GM102, 61-71, 1998.

Holzworth, R. and Meng, C.-I.: Mathematical Representation of the Auroral Oval, Geophys. Res. Lett., 2, 377-380, 1975.

Iijima, T. and Potemra, T. A.: The Amplitude Distribution of FieldAligned Currents at Northern High Latitudes Observed by Triad, J. Geophys. Res., 81, 2165-2174, 1976.

John Hopkins Applied Physics Laboratory: AMPERE, available at: http://ampere.jhuapl.edu/, last access: August 2019.

Kilcommons, L. and Burrell, A. G.: lkilcommons/ssj_auroral_boundary: Version 1 (Version v1.0.0), Zenodo, https://doi.org/10.5281/zenodo.3267415, 2019.

Kilcommons, L. M., Redmon, R. J., and Knipp, D. J.: A new DMSP magnetometer and auroral boundary data set and estimates of field-aligned currents in dynamic auroral boundary coordinates, J. Geophys. Res.-Space, 122, 9068-9079, https://doi.org/10.1002/2016ja023342, 2017. 
Kilcommons, L., Redmon, R., and Knipp, D.: Defense Meteorology Satellite Program (DMSP) Electron Precipitation (SSJ) Auroral Boundaries, 2010-2014 (Version 1.0.0) [Data set], Zenodo, https://doi.org/10.5281/zenodo.3373812, 2019.

Kovalick, T.: SPDF - Coordinated Data Analysis Web (CDAWeb), available at: https://cdaweb.gsfc.nasa.gov, last access: August 2019.

Longden, N., Chisham, G., Freeman, M. P., Abel, G. A., and Sotirelis, T.: Estimating the location of the open-closed magnetic field line boundary from auroral images, Ann. Geophys., 28, 1659-1678, https://doi.org/10.5194/angeo-28-1659-2010, 2010.

Mende, S. B., Heetderks, H., Frey, H. U., Stock, J. M., Lampton, M., Geller, S. P., Abiad, R., Siegmund, O. H. W., Habraken, S., Renotte, E., Jamar, C., Rochus, P., Gérard, J. C., Sigler, R., and Lauche, H.: Far ultraviolet imaging from the IMAGE spacecraft. 3. Spectral imaging of Lyman-alpha and OI $135.6 \mathrm{~nm}$, Space Sci. Rev., 91, 287-381, https://doi.org/10.1007/978-94011-4233-5_10, 2000.

Milan, S.: AMPERE R1/R2 FAC radii, University of Leicester, https://doi.org/10.25392/leicester.data.11294861.v1, 2019.

Milan, S. E., Carter, J. A., Korth, H., and Anderson, B. J.: Principal component analysis of Birkeland currents determined by the Active Magnetosphere and Planetary Electrodynamics Response Experiment, J. Geophys. Res.-Space, 120, 10415-10424, https://doi.org/10.1002/grl.50505, 2015.

More, J., Sorenson, D., Garbow, B., and Hillstrom, K.: The MINPACK Project, in: Sources and Development of Mathematical Software, edited by: Cowell, W., Prentice-Hall, Englewood Cliffs, NJ, USA, available at: https://www.netlib.org/minpack/ (last access: August 2019), 1984.

Newell, P. T., Ruohoniemi, J. M., and Meng, C.-I.: Maps of precipitation by source region, binned by IMF, with inertial convection streamlines, J. Geophys. Res., 109, A10206, https://doi.org/10.1029/2004JA010499, 2004.

Redmon, R. J., Peterson, W. K., Andersson, L., Kihn, E. a., Denig, W. F., Hairston, M., and Coley, R.: Vertical thermal $\mathrm{O}^{+}$flows at $850 \mathrm{~km}$ in dynamic auroral boundary coordinates, J. Geophys. Res., 115, A00J08, https://doi.org/10.1029/2010JA015589, 2010.
Redmon, R. J., Denig, W. F., Kilcommons, L. M., and Knipp, D. J.: New DMSP database of precipitating auroral electrons and ions, J. Geophys. Res.-Space, 122, 9056-9067, https://doi.org/10.1002/2016JA023339, 2017.

Rideout, B.: Madrigal database at CEDAR, available at: http://cedar. openmadrigal.org, last access: August 2019.

Shepherd, S. G.: Altitude-adjusted corrected geomagnetic coordinates: Definition and functional approximations, J. Geophys. Res.-Space, 119, 7501-7521, https://doi.org/10.1002/2014JA020264, 2014.

Spiro, R. W., Heelis, R. A., and Hanson, W. B.: Ion Convection and the Formation and of the Mid-Latitude and F Region Ionization Trough, J. Geophys. Res., 83, 4255-4254, 1978.

Virtanen, P., Gommers, R., Oliphant, T. E., Haberland, M., Reddy, T., Cournapeau, D., Burovski, E., Peterson, P., Weckesser, W., Bright, J., van der Walt, S. J., Brett, M., Wilson, J., Millman, K. J., Mayorov, N., Nelson, A. R. J., Jones, E., Kern, R., Larson, R., Carey, C. J., Polat, İ., Feng, Y., Moore, E. W., VanderPlas, J., Laxalde, D., Perktold, J., Cimrman, R., Henriksen, I., Quintero, E. A., Harris, C. R., Archibald, A. M., Ribeiro, A. H., Pedregosa, F., van Mulbregt, P., and SciPy 1.0 Contributors: SciPy 1.0: Fundamental Algorithms for Scientific Computing in Python, Nat. Methods, in press, 2020.

Waters, C. L., Anderson, B. J., and Liou, K.: Estimation of Global and Field Aligned Currents Using the Iridium System Magnetometer Data, Geophys. Res. Lett., 28, 2165-2168, 2001.

Zhu, Q., Deng, Y., Richmond, A., Maute, A., Chen, Y.J., Hairston, M., Kilcommons, L., Knipp, D., Redmon, R., and Mitchell, E.: Impacts of binning methods on highlatitude electrodynamic forcing: static vs. boundary-oriented binning methods, J. Geophys. Res.-Space, 124, e2019JA027270, https://doi.org/10.1029/2019JA027270, 2019. 